\title{
Performance Evaluation of
}

\section{Incremental Sigma-Delta ADCs Based on their NTF}

\author{
Johannes Wagner, Patrick Vogelmann and Maurits Ortmanns \\ Institute of Microelectronics, University of Ulm, D-89081 Ulm, Germany \\ Email: johannes.wagner@uni-ulm.de
}

\begin{abstract}
In this paper the performance of I-SD ADCs based on the spectral description is discussed. It is based on the modulator and the reconstruction filter and accounts for the applied reset. The definition of the overall noise transfer function (NTF) enables the analysis of the performance of I-SD ADCs in frequency domain and consequently makes it possible to explain the inherent behavior of this type of ADC including reconstruction filter and non-idealities. In the state of the art, the performance of an I-SD ADC is either obtained by simulations or predicted via time-domain considerations. However, these simulations can become time consuming and time-domain considerations neglect non-idealities, especially in the case of a CT modulator. Only recently, a general way to describe the NTF of DT and CT I-SD ADCs was introduced. This work gives a detailed analysis of the NTF of I-SD ADCs and the impact of its properties on the resulting SQNR. It discusses the influence of common reconstruction filters and based on this analysis, it introduces as an example a modified CoI filter that allows to improve the ADC's performance.
\end{abstract}

Index Terms-Incremental, Sigma-Delta, noise transfer function, reconstruction filter.

\section{INTRODUCTION}

In modern signal processing, high performance analog-todigital converters (ADCs) are inevitable as speed and power requirements are becoming more challenging. By trading circuit precision against time, one commonly used type of ADC is the Sigma-Delta (SD) ADC, which is based on oversampling and noise shaping. However, its averaging behavior excludes it from being used in applications where sample-to-sample conversion is required [1]. A solution for this drawback can be the usage of an incremental Sigma-Delta (I-SD) ADC [2]. It provides the benefits of both worlds, the Nyquist-rate behavior and the oversampling and noise shaping capabilities of a conventional SD ADC.

Although I-SD ADCs share the same principles as freerunning SD ADCs, the behavior of an incremental and a freerunning ADC are fundamentally different. Due to the applied periodic reset, the incremental system can not be considered as linear time invariant (LTI) system but rather has to be seen as a linear periodically time variant (LPTV) system. Consequently, the performance analysis of the I-SD ADC in the frequency domain with the noise transfer function (NTF) and the white noise model of the quantizer can not be performed in the same way as for a free-running SD ADC. Moreover, as the reconstruction filter is also reset, it has to be considered and an overall transfer characteristic of the quantization noise has to be determined. As a consequence, the analysis approaches

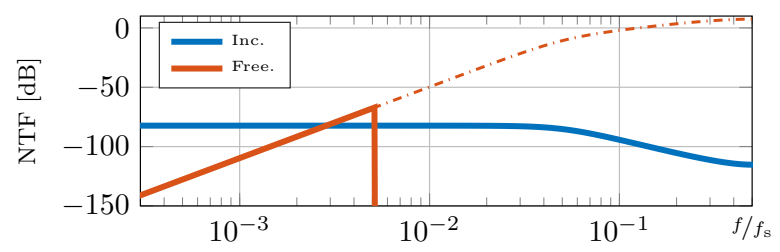

Fig. 1. NTF of an I-SD ADC with a CoI reconstruction filter and NTF of a free-running modulator with an ideal brick-wall filter before decimation. Both are based on the same free-running modulator NTF (dash dotted).

of I-SD ADCs as e.g. in [3], [4] or [5] focus on architecture dependent time-domain considerations. In [6], the same LTI FIR model as in this work is used, however, it only considers ideal, discrete-time (DT) I-SD ADCs, again in the timedomain. Thus, non-ideal behavior, as finite DC gain and finite gain-bandwidth product (GBW) in continuous-time (CT) I-SD ADCs can not be addressed in a straightforward way. In [7], a general way to calculate the overall NTF is described for CT and DT I-SD ADCs. For the CT case, the calculation approach makes use of the lifting method and is able to directly include non-idealities such as excess-loop-delay (ELD), finite DC gain and finite GBW into calculations [8]. In Fig. 1, the overall NTFs (including modulator and subsequent digital filters) of a free-running SD ADC and an incremental SD ADC before decimation are shown, illustrating the difference of both: whereas the NTF of the free-running Sigma-Delta modulator (SDM) is only considered until the bandedge, where the digital decimation filter will ideally cut off all out-ofband (OOB) components, the performance of the I-SD ADC is heavily determined by the OOB components.

Whereas the general approach to determine the overall NTF was derived in [7], there was no in-depth analysis on its behavior. Therefore, this work focuses on the properties of the overall NTF of I-SD ADCs, which has, to the authors knowledge, not been covered in literature so far. As the signalto-quantization-noise ratio (SQNR) can be estimated based on the NTF, the performance of I-SD ADCs is analyzed alongside. The estimate makes time-consuming simulations redundant and allows a systematic system design approach. Consequently, the influence on the ADC's performance of common reconstruction filters and of the modulator with applied reset are discussed. Based on these findings, this work exemplarily introduces a modified chain-of-integrators (CoI) filter that allows to improve the ADC's performance in order to emphasize the significance of this analysis.

Section II discusses the working principle of I-SD ADCs 

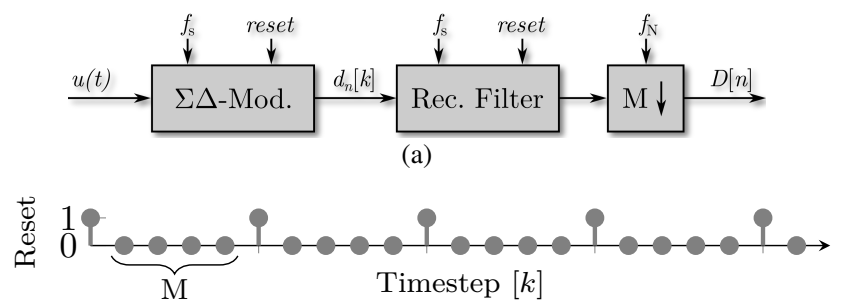

(b)

Fig. 2. Basic block diagram of an I-SD ADC (a) and the corresponding rese diagram for an OSR of $M+1$ (b).

in time domain. The calculation of the overall NTF of I-SD ADCs and its differences to the free-running counterpart is reviewed in Section III. In Section IV, properties of the NTF depending on the modulator and the reconstruction filter are discussed. Based on this analysis, in Section V an improved version of a CoI filter is given. Section VI concludes the paper.

\section{WORKING PRINCIPLE OF I-SD ADCS}

In order to give a first overview of an I-SD ADC, the working principle of a first order ADC is introduced in time domain as commonly described in literature [1][3]. In general, an I-SD ADC as in Fig. 2a consists of the incremental SigmaDelta modulator (I-SDM), the digital reconstruction filter and the decimator. In the DT case, the continuously running input signal $u(t)$ is sampled at the integrator input resulting in samples $u_{n}[k]$. Here $n$ denotes the $n$-th Nyquist-rate sample and $k=1 \ldots M$ is the running variable of the oversampled modulator, where $M$ samples at $f_{\mathrm{s}}$ - the oversampling rate are used to generate one Nyquist sample at $f_{\mathrm{N}}$. In I-SDM, $M$ is equal to the oversampling ratio (OSR) for an infinitely short reset. Consequently, the integrator output is calculated in time domain by

$$
y_{n}[k]=\sum_{l=1}^{k}\left(u_{n}[l]-d_{n}[l]\right) .
$$

The basic difference of an I-SDM to a conventional SDM is its periodically applied reset window as shown in Fig. 2b. In all further considerations, a linearized model is used where the quantizer is replaced by a noise source $e[k]$ and the linear gain $q$. Therefore, the I-SDM generates $M$ samples $d_{n}[k]$ for $k \in$ $[1, M]$ with $d_{n}[k]=q \cdot y_{n}[k]+e[k]$, which the reconstruction filter and the subsequent decimator use to create one sample

$$
D[n]=\frac{1}{M} \sum_{l=1}^{M} d_{n}[l] .
$$

The output $D[n]$ yields the digital representation of the analog input at Nyquist-rate $f_{\mathrm{N}}$. Therefore, each conversion cycle is independent from each other and true Nyquist ADC behavior is obtained. Based on the difference of the digital output and the input, the conversion error and thus the performance of the converter can be estimated. However, these calculations are architecture specific and often require assumptions and simplifications. This is especially true for CT I-SDMs as their specific behavior and non-idealities are usually neglected. Additionally, an upfront sample-and-hold ( $\mathrm{S} / \mathrm{H})$ element is assumed, although not always present in the actual design [4].

\section{OVERALL NTF OF I-SD ADCS}

In Section II and in most prior literature, it is shown that I-SD ADCs can be described in time domain. Nonetheless, a description in frequency domain is often desired, which can be used especially in the CT case to include non-idealities. Therefore, in this chapter the description of an I-SD ADC in the frequency domain - as introduced in [7] - is shortly reviewed. In the following a normalized sampling frequency $f_{\mathrm{s}}=1$ is assumed for simplicity and readability of the analysis. The NTF of a conventional SDM is usually obtained with the help of the Z-transform by using the LTI system model. However, in the overall transfer characteristic for I-SD ADCs also the reconstruction filter and the time-variant behavior due to the reset have to be considered, resulting in a modified definition of the NTF: it describes the transfer characteristic of quantization noise introduced by the quantizer to the output of the reconstruction filter. For a free-running SD ADC, this ideally corresponds to the multiplication of the NTF with a brick-wall filter, as illustrated in Fig. 1. After the downsampling, all components of the overall NTF above $f_{\mathrm{N}}$ are folded back in the frequency range between 0 and $f_{\mathrm{N}}$, usually resulting in a flat spectrum seen from I-SD ADCs.

\section{A. Lifting Method for CT I-SD ADCs}

Due to the mixed signal character of the CT SDM, the calculation of the NTF is not straightforward. In order to derive a NTF, an equivalent DT model has to be obtained. For this purpose, in [7] the lifting method is used [8]. In [9] CT SDMs with arbitrary feedback digital-to-analog converters (DACs) and the CT filter function $H_{C T}(s)$ including non-idealities such as finite DC gain and GBW - can be transformed by the lifting method to a DT filter $H(z)$. The analytically derived, equivalent DT model behaves exactly as the CT model at the sampling instants and serves as basis for the incremental NTF in the same way as the filter of a genuine DT ADC.

\section{B. NTF Calculation}

The applied reset alters the system characteristics of an I-SD ADCs from an LTI to an LPTV system. However, it has been shown that I-SD ADCs can be modeled as a LTI system finite impulse response (FIR) [10], [7]. With the DT filter $H(z)$ or the DT equivalent of the CT filter, the NTF of the free-running SDM can be expressed by

$$
N T F_{\mathrm{sdm}}(z)=\frac{1}{1+q \cdot H(z)} .
$$

The Z-transform of the overall transfer function of a freerunning DT $\mathrm{SD} \mathrm{ADC}, N T F_{\text {free}}$, is given by

$$
N T F_{\text {free }}(z)=N T F_{\mathrm{sdm}}(z) \cdot H_{\text {rec }}(z)
$$

with $H_{\mathrm{rec}}(z)$ being the transfer function of the reconstruction filter. In time domain, this yields an infinite convolution sum with the respective impulse responses of the two subsequent systems. Without reset, also the impulse response $h_{\text {free }}[n]$ of the ADC would be infinite due to the infinite impulse response $h_{\mathrm{sdm}}[n]$ of the modulator. However, it is proven in [10] that by 
TABLE I

DifFERENCE OF CALCULATED AND MATLAB SIMULATED SQNRS OF THE ADCS WITH DIFFERENT GBW AND DC GAIN COMBINATIONS AT $A_{\text {in }}=-10 \mathrm{dBFS}$ AND $f_{\text {in }}=0.5 f_{\mathrm{N}}$ FOR $M=100$.

\begin{tabular}{cccc}
\hline GBW $\left[f_{\mathrm{s}}\right] / \mathrm{DC}$ Gain & $10 / 1000$ & $5 / 100$ & $1 / 20$ \\
\hline Single-Bit: & $0.1 \mathrm{~dB}$ & $0.1 \mathrm{~dB}$ & $1.3 \mathrm{~dB}$ \\
Multi-Bit: & $0.6 \mathrm{~dB}$ & $0.5 \mathrm{~dB}$ & $0.4 \mathrm{~dB}$ \\
\hline
\end{tabular}

truncating the infinite convolution sum, the impulse response of an equivalent FIR filter

$$
\begin{aligned}
h_{\mathrm{FIR}}[k] & =\sum_{l=0}^{M-1} h_{\mathrm{sdm}}[k-l] \cdot h_{\mathrm{rec}}[l] \\
& =\mathcal{Z}^{-1}\left\{N T F_{\text {free }}(z)\right\}[k] \cdot \operatorname{rect}_{M}[k] .
\end{aligned}
$$

can be obtained for $k \in[0, M-1]$, which accounts for the reset. The expression $\operatorname{rect}_{M}[k]$ is a DT rectangular window function with length $M$ and $N T F_{\text {free }}(z)$ is the combination of the NTF of the free-running SDM and the reconstruction filter transfer function as defined in (4). In the frequency domain, this calculation corresponds to the circular convolution of the overall free-running NTF

$$
N T F_{\text {incr }}(f)=N T F_{\text {free }}(f) \circledast X_{\text {rect }, M}(f)
$$

with the DT sinc function

$$
X_{\text {rect }, M}(f)=\frac{\sin (M \pi f)}{\sin (\pi f)} \cdot e^{-j(M-1) \pi f}
$$

by applying the discrete time Fourier transform (DTFT) as shown in detail in [7]. As it can be seen in the following sections, the inclusion of the reconstruction filter and the convolution with the DT sinc have major influence of the characteristics of the incremental ADC.

\section{Prediction of the SQNR based on the NTF}

The overall NTF of an I-SD ADC allows to directly estimate the SQNR of I-SD ADCs in combination with the white noise model in a general way, comparable to its free-running counterpart. The estimated SQNR is determined by

$$
S Q N R_{\mathrm{est}}=10 \cdot \log _{10}\left(\frac{P_{\mathrm{in}}}{I B N_{\mathrm{est}}}\right)
$$

with integrated inband noise

$$
I B N_{\mathrm{est}}=\frac{\Delta^{2}}{12} \cdot \int_{-0.5}^{0.5}\left|N T F_{\mathrm{incr}}(f)\right|^{2} d f,
$$

the signal power $P_{\text {in }}$ and the quantizer step size $\Delta$ [1]. Due to the accuracy of the lifted CT models, also the influence of non-idealities such as finite GBW, finite DC gain and ELD are covered precisely for CT ADCs. This allows an analytic prediction of the ADC's performance without simulation.

Table I shows exemplarily the accuracy of the calculated SQNR as in (8) for a CT ADC in cascade-of-integrators feedforward (CIFF) architecture with different GBW and DC gain combinations. A single-bit and a multi-bit (4-bit) quantizer are used at an OSR of 100 based on $2^{12}$ samples. The simulation and calculation match closely in the range of the applicability of the white noise model and numeric variations. Consequently, the following analysis is based on the overall NTF and (8).

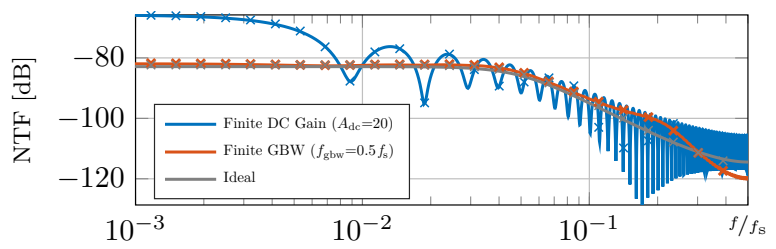

Fig. 3. Calculated overall NTFs of an I-SD ADC utilizing integrators with finite GBW or finite DC gain in comparison to the ideal case with annotated Matlab simulation results.

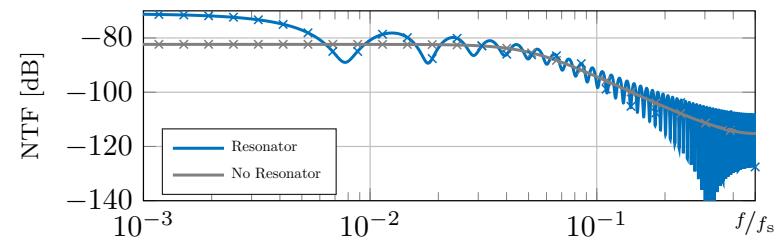

(a)

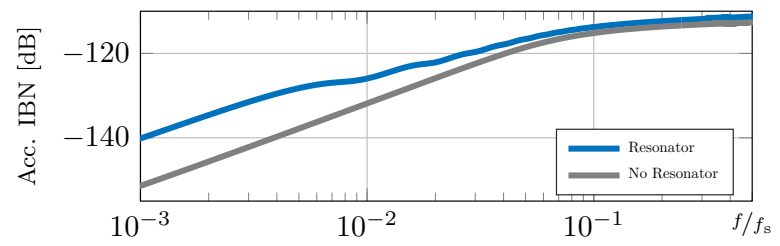

(b)

Fig. 4. Calculated overall NTF with annotated Matlab simulation results (a) and accumulated IBN up to a certain frequency (b) of an I-SD ADC with optimized zeros in comparison to a corresponding ADC without them.

\section{Performance AnAlysis of I-SD ADCs BASED ON THE OVERALL NTF}

Based on the applicability of the frequency-domain description and using the NTF derived for I-SD ADCs, in this section, their performance is discussed on system level in comparison to their free-running counterparts. In contrast to the state of the art, in this work, this is done - to the authors knowledge for the first time - in the frequency domain. As reference, the corresponding free-running SD ADCs are utilized, as, except for very low OSRs, the performance of I-SD ADCs is comparatively lower [4]. Consequently, it must be the goal to reduce this difference.

For free-running SD ADCs, usually only the modulator part is analyzed and for the decimation filter, an ideal brick-wall filter is assumed. The SDM predominantly determines the ADC's performance and is therefore at the center of attention. In the following, the influence mechanisms onto I-SD ADCs are analyzed separately for the I-SDM and the digital filter. For the analysis, coefficients for a third-order DT modulator are obtained by the Delta-Sigma Toolbox with an out-of-band gain (OBG) of 1.5 and internal states scaled to 0.9 full-scale. For its CT counterpart, an impulse invariant transform is performed. If not stated otherwise, an OSR of 100 is used and for the incremental case, a digital filter of the same order is utilized.

\section{A. Influence of the I-SD Modulator}

1) Modulator with Finite DC Gain and GBW: Major nonidealities in CT SDMs are the finite GBW and the finite DC gain of the integrators. Both alter the shape of the overall NTF of an I-SD ADC and - as presented in Section III-C 
- the individual influence on the SQNR can by precisely predicted and analyzed in comparison to the free-running case. An example is illustrated in Fig. 3: alongside the ideal overall NTF, the NTFs with either applied finite GBW or DC gain are presented. Simulation results are annotated to underline the accuracy of the overall NTFs comprising non-idealities. It can be seen, that for finite GBW, the overall NTF shape is altered mainly in the higher frequency region, which is attenuated by the digital reconstruction filter. In the free-running case, these changes are completely suppressed due to the brickwall filter, but are known to yield stability problems to some extent. In the lower frequency region, the shape is only slightly elevated resulting in a relatively small drop of SQNR of $0.5 \mathrm{~dB}$ in the incremental case and $1.0 \mathrm{~dB}$ in the free-running case for $f_{\mathrm{gbw}}=0.5 f_{\mathrm{s}}$. In contrast, finite DC gain influences the frequency components closer to DC. In the case of the freerunning NTF, the inband close to DC is known to flatten by finite DC gain, resulting in an increased IBN of $3.0 \mathrm{~dB}$ for $A_{\mathrm{dc}}=20$. For the I-SD ADC, however, the IBN increases by even $6.0 \mathrm{~dB}$. The overall NTF gives the reason: it exposes the characteristic of a sinc function with notches in proximity to multiples of $1 / M$ and an amplified main lobe caused by the circular convolution with $X_{\text {rect, } M}(f)$.

2) Modulator with Optimized Zeros: The incremental operation also influences techniques, which are commonly used to improve the SQNR for free-running ADCs, however, have negative influence on incremental ADCs. For free-running SD ADCs, the optimization of the NTF zeros is usually employed [1]. By analyzing the overall NTF, it can be explained why this method is not beneficial in the incremental case. In Fig. 4a, the exemplary overall NTFs with and without optimized zeros are depicted alongside with annotated simulation results.

For the free-running NTF, the optimized zeros not only introduce a notch but also elevate the frequency components close to DC [1]. Similar as in the case of finite GBW, this raise of the free-running NTF close to DC in combination with the circular convolution exposes a sinc-like shape of the overall NTF of the I-SD ADC. In Fig. 4b the accumulated IBN up to a certain frequency is shown for the case with and without optimized zeros. It can be clearly seen that the increased IBN originates from the main lobe of the sinc-like function. Both cases approach each other for higher frequencies as the subsequent notches attenuate certain noise components - still, a small loss in performance can be observed in total.

\section{B. Influence of the Reconstruction Filter}

As motivated above, the I-SD ADC needs to be analyzed as a whole. Therefore, in this section the reconstruction filter is taken into account.

1) Chain-of-Integrators Filter: Due to its simplicity while still providing a good performance, the CoI reconstruction filter is often used in I-SD ADC implementations. It consists of a chain of digital integrators, commonly of the same order as the analog modulator [1]. In literature also of one order higher is mentioned [3][11]. However, this is not analyzed in general and only in time domain for DC measurement.

Consequently, this section analyses both options referenced to the free-running counterpart. In Fig. 5a the difference of the

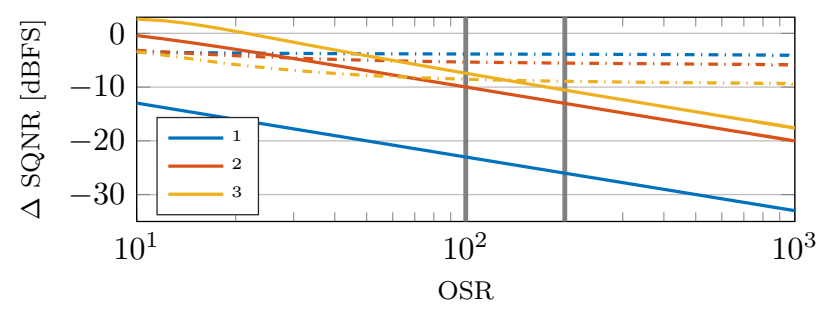

(a)

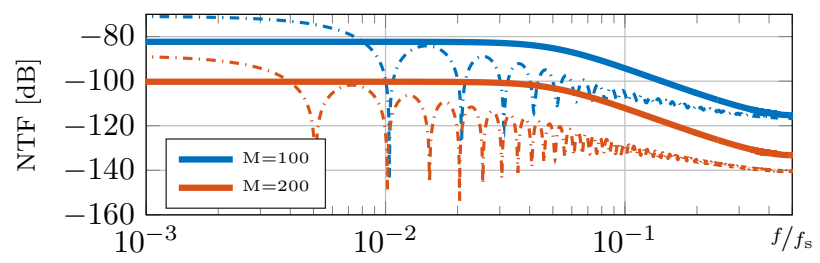

(b)

Fig. 5. Calculated $\triangle$ SQNR of I-SD ADCs and a free-running ADCs from first to third order with a CoI filter of the same order (solid) and one order more (dash-dotted) (a) and exemplary NTFs of a 3rd order ADC (b).

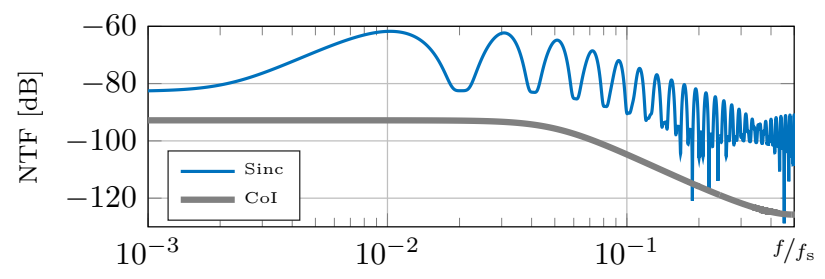

Fig. 6. Calculated overall NTFs of an 3rd order I-SD ADC with a 4th order sinc in comparison to a 3rd order CoI at an OSR of 100.

SQNR predictions of I-SD ADCs to free-running SD ADCs with respect to the OSRs is shown. Modulators from first to third order are combined with a reconstruction filter of the same order (solid) or one order more (dash-dotted). Fig. 5a shows that for a CoI of the same order as the analog modulator, the difference to its free-running counterpart increases with the OSR. With a CoI filter of one order higher than the modulator order, for a first and second order modulator, the I-SD ADC can achieve a performance much closer to the one of the freerunning counterpart. For higher order modulators, however, again an increased SQNR difference can be noticed. Though, it can be noticed that the difference, in contrast to the $\mathrm{CoI}$ at the same order, is rather constant for higher OSR.

In Fig. 5b the corresponding overall NTFs are shown for $M=100$ and $M=200$ for a third-order modulator and both CoI variants. The I-SD ADC with the CoI reconstruction filter of one order higher shows the sinc like shape similar as for modulator with optimized zeros. However, for the case of $M=200$, the NTF shows a higher attenuation in higher frequency regions. Moreover, as the main lobe width varies with the OSR, the accumulated IBN reduces proportionally, leading to the constant performance difference with the freerunning ADC due to its also OSR dependent brick-wall filter.

2) Sinc Filters: Another type of reconstruction filter often mentioned in literature is the sinc or triangular filter. As presented in [12], it allows line noise suppression due to the possibility to add notches to the overall signal transfer function (STF) of the ADC. For an exemplary decimation factor of $N=50$ and $M=150$, this leads to deep notches in the 
STF at multiples of $0.02 f_{\mathrm{s}}$. However, this property comes along with the drawback of a decreased SQNR, which can be explained with the overall NTF as in Fig. 6. Here, the NTF of a third-order ADC utilizing a sinc filter is illustrated in comparison with a CoI filter based ADC. The NTF shows an overall amplification and notches at DC and at the same frequencies as for the STF. Additionally, these notches are filled up by the circular convolution due to the reset.

\section{MOdified CoI FILTER}

In Section IV, the influence of different reconstruction filters on the SQNR has been presented. The ADC utilizing a CoI filter with one order higher than the modulator can outperform a filter at the same order for higher OSR; however, for e.g. a third-order modulator, for lower OSRs, the filter at the same order outperforms it. The conclusion can be drawn that the filter needs to be adapted to the OSR of the incremental ADC. Therefore, in this section, in order to increase the SQNR for medium OSRs, a modified CoI is introduced based on the previous analysis of the overall NTF.

\section{A. Transfer Function}

In Fig. $5 b$ it can be seen that a certain OSR is required to make the ADC benefit from the increased CoI order. If a certain threshold is exceeded, the increased power in the main lobe is compensated by the reduced power for higher frequencies. In order to modify this behavior, the structure of the CoI filter can be adapted. In Fig. 7, a modified fourth order CoI filter is shown that can be utilized for a third-order modulator. One of the integrators has an adjusted feedback gain of $h=0.96$ that modifies the NTF as in Fig. 8b. The modified feedback results in reduced power for low frequencies compared to a conventional $\mathrm{CoI}$ filter at the same order, and a steeper roll off for higher frequencies, while the notches are vanishing. This compromises the properties of the third and fourth order CoI filters.

\section{B. Performance Evaluation}

Based on the overall NTF, again, the performance with respect to the OSR can be analyzed. The modified structure allows a performance gain compared to both the CoI filter at the same order and one order increased as can be seen exemplarily in Fig. 8a. It can be seen that the modified structure is beneficial for an OSR between around 60 and 700 . A performance increase of up to $6.9 \mathrm{~dB}$ can be achieved for $M=700$ where the fourth order CoI starts to outperform the modified CoI filter.

\section{CONCLUSION}

In this work an analysis of the overall NTF of I-SD ADCs and the impact of its properties on the resulting SQNR is given. The work is based on a general way to calculate the overall NTF of DT and CT I-SD ADCs. The overall NTF allows to directly draw conclusions on the ADC's performance including non-idealities. Moreover, the behavior of common reconstruction filters as $\mathrm{CoI}$ and sinc filters is discussed. Based on this analysis, a modified CoI filter that allows to improve the performance ADCs for medium OSRs is introduced.

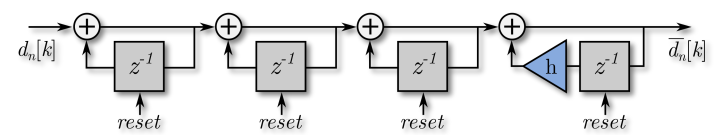

Fig. 7. 4th order modified CoI filter comprising one lossy integrator.

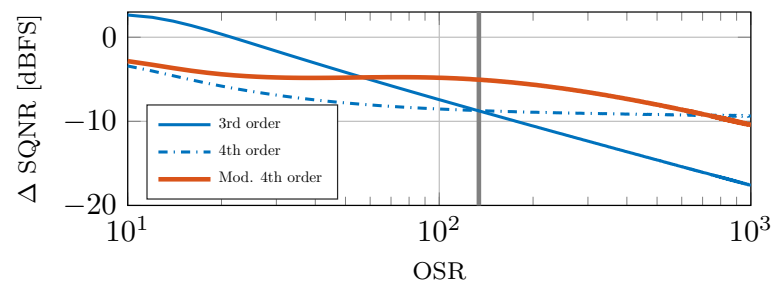

(a)

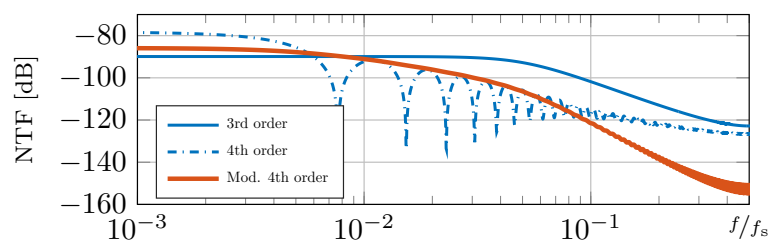

(b)

Fig. 8. Calculated SQNR difference of a 3rd order I-SD ADC combined with a 3rd order, a 4th order CoIs filter and the modified CoIs filter and a free-running SD ADC (a) and exemplary NTFs at an OSR of 100.

\section{ACKNOWLEDGMENT}

This work was supported by German Research Foundation under DFG grant OR 245/10-1.

\section{REFERENCES}

[1] S. Pavan, R. Schreier, and G. C. Temes, Understanding Delta-Sigma Data Converters., 2nd ed. Wiley-IEEE Press, 2017.

[2] R. van de Plassche and R. E. J. van Der Grift, "A five-digit analogdigital converter," IEEE Journal of Solid-State Circuits, vol. 12, no. 6, pp. 656-662, Dec. 1977.

[3] J. Markus, J. Silva, and G. C. Temes, "Theory and applications of incremental $\Delta \Sigma$ converters," IEEE Transactions on Circuits and Systems I: Regular Papers, vol. 51, no. 4, pp. 678-690, Apr. 2004.

[4] T. C. Caldwell and D. A. Johns, "Incremental data converters at low oversampling ratios," IEEE Transactions on Circuits and Systems I: Regular Papers, vol. 57, no. 7, pp. 1525-1537, Jul. 2010.

[5] P. Vogelmann, J. Wagner, M. Haas, and M. Ortmanns, "A dynamic power reduction technique for incremental modulators," IEEE Journal of SolidState Circuits, pp. 1-13, 2019.

[6] J. Steensgaard, Z. Zhang, W. Yu, A. Sarhegyi, L. Lucchese, D. I. Kim, and G. C. Temes, "Noise - power optimization of incremental data converters," IEEE Transactions on Circuits and Systems I: Regular Papers, vol. 55, no. 5, pp. 1289-1296, Jun. 2008.

[7] J. Wagner, P. Vogelmann, and M. Ortmanns, "On the signal filtering property of CT incremental sigma-delta ADCs," IEEE Transactions on Circuits and Systems II: Express Briefs, p. 1, 2019.

[8] M. Keller, A. Buhmann, M. Ortmanns, and Y. Manoli, "A method for the discrete-time simulation of continuous-time sigma-delta modulators," in 2007 IEEE International Symposium on Circuits and Systems, May 2007, pp. 241-244.

[9] T. Brückner, M. Kiebler, M. Lorenz, C. Zorn, W. Mathis, and M. Ortmanns, "Calculating transfer functions of CT sigma-delta modulators with arbitrary DAC waveforms," in Proc. IEEE Int. Symp. Circuits and Systems (ISCAS2013), May 2013, pp. 1264-1267.

[10] S. Bannwarth, A. Wenzler, and W. Mathis, "On the transfer behaviour of incremental sigma delta converters," in Proc. VXV Int. Symp. Theoretical Engineering, Jun. 2009, pp. 1-5.

[11] J. Robert and P. Deval, "A second-order high-resolution incremental A/D converter with offset and charge injection compensation," IEEE Journal of Solid-State Circuits, vol. 23, no. 3, pp. 736-741, Jun. 1988.

[12] J. Markus, P. Deval, V. Quiquempoix, J. Silva, and G. C. Temes, "Incremental delta-sigma structures for DC measurement: an overview," in Proc. IEEE Custom Integrated Circuits Conf. 2006, 2006, pp. 41-48. 